\section{Use of transcatheter contrast-enhanced ultrasound with vessel navigation to reduce the contrast requirement during transarterial chemoembolization in patients with renal impairment}

Uei Pua ${ }^{1,2}$

${ }^{1}$ Department of Diagnostic Radiology, Tan Tock Seng Hospital, ${ }^{2}$ Yong Loo Lin School of Medicine, National University of Singapore, Singapore

Modifications to the transarterial chemoembolization (TACE) technique are often required in patients with preexisting renal impairments, but the literature surrounding this scenario is scarce. A 77-yearold man with moderate renal impairment presented with a 7.8- $\mathrm{cm}$ hepatocellular carcinoma in the right hepatic lobe. His renal function deteriorated after a single session of portal vein embolization and three sessions of TACE, with his estimated glomerular filtration rate declining from 29 to $12 \mathrm{~mL} /$ $\min / 1.73 \mathrm{~m}^{2}$. Follow-up noncontrast magnetic resonance imaging showed a partial response with cystic changes in the tumor and residual tumor in the posterior aspect (Supplementary Fig. 1A), and an additional session of TACE was required.

For the fourth session of TACE, we modified our technique to use a combination of software navigation and transcatheter contrast-enhanced ultrasound (CEUS) to complete the TACE with only a single injection of contrast agent. For catheter placement, after cannulation of the celiac axis, dual-phase cone-beam computed tomography (CBCT) (XperCT Dual, Philips Medical Systems, Best, Netherlands) was performed using a single bolus of $12 \mathrm{~mL}$ of contrast injected at $6 \mathrm{~mL} / \mathrm{sec}$ through a power injector. Post-processing was performed using the EmboGuide vessel navigation software (Philips Medical Systems) to identify the feeding vessel and to guide the 2.3-F microcatheter (Supplementary Fig. 1B) to the location. To confirm the correct catheter position for embolic delivery, a second operator positioned an ultrasound (US) probe over the region of the residual tumor. US contrast (SonoVue, Bracco, Milan, Italy) was then hand-injected through the microcatheter at a 1:3 dilution (2.4 mL of SonoVue diluted to $10 \mathrm{~mL}$ with normal saline). The tumor was observed realtime for 30 seconds using imaging protocols similar to intravenous CEUS examinations (e.g., a low mechanical index of $<0.08$ ). Satisfactory catheter position was confirmed by visualizing avid tumor enhancement (Supplementary Fig. 1C, Video clip 1), followed by the infusion of $3 \mathrm{~mL}$ of Oncozene (Boston Scientific, Natick, MA, USA) and $100 \mathrm{mg}$ of doxorubicin mixed with $5 \mathrm{~mL}$ of contrast and 5 $\mathrm{mL}$ of normal saline. Good tumoral uptake of Oncozene was immediately seen on CBCT (Supplementary Fig. 1D) and complete stasis was demonstrated with the injection of a second bolus of SonoVue. The total iodinated contrast volume was $17 \mathrm{~mL}$, including the $5 \mathrm{~mL}$ in the embolic mixture. The patient's creatinine level remained stable immediately after the procedure, and follow-up CEUS at 6 weeks confirmed a complete response.

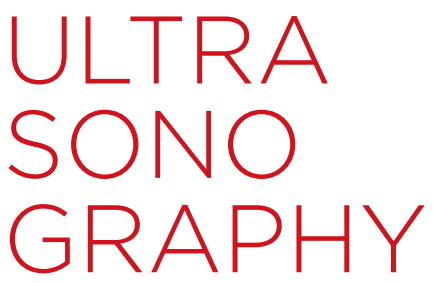

\section{LETTER}

https://doi.org/10.14366/usg. 17006 pISSN: 2288-5919 e elSSN: 2288-5943 Ultrasonography 2017;36:284-285

Received: January 30, 2017

Revised: March 5, 2017

Accepted: March 13, 2017

Correspondence to: Uei Pua, MBBS, MMed, FRCR, FAMS, Department of Diagnostic Radiology, Tan Tock Seng Hospital, 11 Jalan Tan Tock Seng, Singapore 309199, Singapore

Tel. +65-81819490

Fax. +65-67599940

E-mail: druei@yahoo.com

This is an Open Access article distributed under the terms of the Creative Commons Attribution NonCommercial License (http://creativecommons.org/ licenses/by-nc/3.0/) which permits unrestricted noncommercial use, distribution, and reproduction in any medium, provided the original work is properly cited.

Copyright (C) 2017 Korean Society of Ultrasound in Medicine (KSUM)

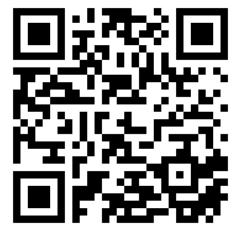

How to cite this article:

Pua U. Use of transcatheter contrastenhanced ultrasound with vessel navigation to reduce the contrast requirement during transarterial chemoembolization in patients with renal impairment. Ultrasonography. 2017 Jul;36(3):284-285. 
Novel intra-arterial sonographic techniques, such as carbon dioxide or microbubbles that are virtually non-nephrotoxic, are attractive alternatives to TACE. However, disadvantages of carbon dioxide, such as persistent staining and difficulty in the identification of hyperechoic lesions, have been discussed [1]. The use of intraarterial CEUS has previously been shown to be safe and feasible, with the ability to improve superselectivity during TACE in small studies $[2,3]$. The use of intra-arterial CEUS as an alternative contrast agent during TACE to replace conventional angiography and to demonstrate "tumor blush" with microbubble perfusion in patients with renal impairment has not been described, although it is at least anecdotally feasible. In combination with the use of advanced CBCT techniques, the total iodine burden could be significantly lowered. Thus, the development of a zero-iodine-contrast TACE technique specifically in patients with renal impairment could be an area of interest and future development.

ORCID: Uei Pua: http://orcid.org/0000-0001-8896-5648

\section{Conflict of Interest}

No potential conflict of interest relevant to this article was reported.

\section{Supplementary Material}

Supplementary Fig. 1. A 77-year-old man with moderate renal impairment presented with a 7.8-cm hepatocellular carcinoma in the right hepatic lobe. A. T2-weighted axial magnetic resonance imaging after three sessions of transarterial chemoembolization (TACE) shows cystic changes in a large part of the tumor, consistent with tumor necrosis. However, residual viable tumor (asterisk) was noted in the posterior aspect of the tumor, which needed further TACE. His estimated glomerular filtration rate declined from 25 to $12 \mathrm{~mL} / \mathrm{min} / 1.73 \mathrm{~m}^{2}$ over the three TACE sessions. B. Fluoroscopic image of the position of the microcatheter prior to contrastenhanced ultrasound (CEUS) injection is shown. The microcatheter was guided based on the best angulation of the C-arm (right anterior oblique $63^{\circ}$, caudal tilt $7^{\circ}$ ) as determined by the navigation software (arrowheads). The tumor was selected manually after dualphase computed tomography (CT) on the workstation and depicted as blue on the display. The same path was precisely replicated using a microcatheter. This was the position of the intra-arterial contrast agent injection for CEUS. C. CEUS obtained 15 seconds after the transcatheter injection of SonoVue (right) shows brisk contrast enhancement in the viable tumor (asterisk), with the nonviable tumor showing no uptake of microbubbles $(x)$ compared to the real-time grey-scale reference image (left). Enhancement of the pseudocapsule (arrowheads) around the tumor is also present and well demonstrated. Embolization was performed from this catheter position based on these findings. D. A noncontrast cone-beam CT image obtained immediately after embolization shows good uptake of embolic materials in the targeted residual tumor, consistent with technical success (https//doi.org/10.14366/usg.17006).

Video clip 1. Real-time video clip of ultrasound after the transcatheter injection of SonoVue. Brisk contrast enhancement in the viable tumor is well defined, based on the nonviable tumor portion showing no uptake of microbubbles, combined with delayed enhancement of the tumor pseudocapsule, as indicated in Supplementary Fig. 1C. Embolization was performed from this catheter position based on these findings (https://doi.org/10.14366/ usg. 17006.v001).

\section{References}

1. Pereira K, Salsamendi J, Puchferran C. Use of intrarterial carbon dioxide-enhanced ultrasonography (COEUS) in patient with renal insufficiency undergoing trans arterial-chemo-embolization (TACE). Cardiovasc Intervent Radiol 2016;39:957-959.

2. Burgmans MC, van Erkel AR, Too CW, Coenraad M, Lo RH, Tan BS. Pilot study evaluating catheter-directed contrast-enhanced ultrasound compared to catheter-directed computed tomography arteriography as adjuncts to digital subtraction angiography to guide transarterial chemoembolization. Clin Radiol 2014;69:10561061.

3. Moschouris H, Malagari K, Kalokairinou M, Stamatiou K, Marinis A, Papadaki MG. Contrast-enhanced ultrasonography with intraarterial administration of SonoVue for guidance of transarterial chemoembolization: an initial experience. Med Ultrason 2011;13:296-301. 